\title{
Primary pulmonary nodular Amyloidosis
}

\author{
F. Agresta1, A. Marin2, D. Della Libera33, F. Romanzi4, L.F. Ciardo1, \\ L. Bittesini3, S. Nardini2, N. Bedin 1
}

ABSTRACT: Primary pulmonary nodular Amyloidosis. F. Agresta, A. Marin, D. Della Libera, F. Romanzi, L.F. Ciardo, L. Bittesini, S. Nardini, N. Bedin.

Primary nodular amyloidosis of the lung is an uncommon manifestation. The disease runs a benign course, but offers diagnostic problems due to non-specific radiological features entering the big field of the solitary nod- ule. We describe the case of a 60 year old man with multiple nodules on the left lung operated on diagnostic and therapeutic video-assisted thoracoscopy and discuss the possibilities, if any, of suspecting such a disease through radiologic characteristics along with findings from the patient's history, physical examination and laboratory tests. Monaldi Arch Chest Dis 2005; 63: 3, 173-175.

Keywords: Solitary pulmonary nodule; Amyloidosis; Amyloidoma; Differential diagnosis.

1 Dept. of General Surgery, ASL 7 della Regione Veneto. Presidio Ospedaliero di Vittorio Veneto (TV)

2 Dept. of Pneumology, ASL 7 della Regione Veneto. Presidio Ospedaliero di Vittorio Veneto (TV)

3 Dept. of Histopathology, ASL 7 della Regione Veneto. Presidio Ospedaliero di Conegliano Veneto (TV)

4 Dept. of Radiology, ASL 7 della Regione Veneto. Presidio Ospedaliero di Vittorio Veneto (TV). Italy.

Correspondence: F. Agresta, M.D., Dept. of General Surgery, ASL 7 della Regione Veneto, Presidio Ospedaliero di Vittorio Veneto, Via Forlanini 71, 31029 Vittorio Veneto (TV), Italy; e-mail: fagresta@libero.it

\section{Introduction}

Nodular amyloid lesions of the lung are usually a localised form of amyloidosis [1-2-3]. Thus far, no typical radiological findings have been described to distinguish pulmonary amyloidosis from lung cancer, metastatic or granulomatous diseases, tuberculosis, silicosis, sarcoidosis and hamartoma [1-2-3-4]. Moreover, the hard consistency of amyloid tissue makes it difficult to obtain biopsy specimens and to diagnose amyloid lesion of the lung by CT-guided lung biopsy (transbronchial and or percutaneous). As a result, the majority of cases of pulmonary amyloidosis have been diagnosed only through thoracotomy [5-6-7-8-9].

This report presents a case of pulmonary amyloidosis diagnosed by video-assisted thoracoscopic biopsy, which was, in the same time, diagnostic and therapeutic.

\section{Case Report}

A 60 year-old man presented with multiple left lung nodules (upper lingula's segment and dorsal segment of upper lobe) accidentally discovered, on a chest-X-Ray (with no calcification and not detected in previous radiographs). No other complaints were reported and no clinical signs were revealed during physical examination. He was a heavy smoker (40 cigarettes per day for more than 40 years) and he was under medical treatment, for a benign hypertension, with an ACE-inhibitor. He was obese $(100 \mathrm{Kg}$ and $170 \mathrm{~cm} \mathrm{~h})$ and had history of gout medically treated with allopurinol, and a surgical intervention for a peritonitis has been per- formed due to an acute appendicitis. No other relevant medical history or drugs abuse was present. Laboratory tests including complete blood count, serum chemistry, tumour markers and arterial blood gases revealed normal results. The patient's serum analysis was normal. No Bence-Jones protein was found in $24 \mathrm{~h}$ urine collections. Pulmonary function tests showed slight airway obstruction with normal $\mathrm{CO}$ diffusion. Bronchoscopy revealed a normal tracheobronchial tree. Bronchoalveolar lavage was negative for malignancy. On CT scan the nodules were located on the upper segment of the lingula and in the dorsal segment of the upper lobe. With a subpleurical extrication and light hyperdensity after contrast medium, about $3 \mathrm{~cm}$ in diameter each and with irregular edge contours (fig. 1). Transthoracic fine-needle aspiration (CT-guided) of pulmonary nodules was negative. Globally considering the patient (his age, history - heavy smoker, the radiologic features, and the important risk of lung cancer), with an obtained informed consensus, a Video-Assisted-ThoracoScopy lung biopsy (with a wedge resection of both the nodules - three trocars approach) has been carried out with an uneventful post-operative period.

Macroscopically, the nodules, which were on the surface of the lung, were about $3 \mathrm{~cm}$ in diameter, hard in terms of consistency and semi translucent grey in colour. Histologically, the specimens contained acidophilic homogeneous material with foreign body giant cells, histiocytes and fibroblasts and areas of osteo-cartilaginoid metaplasia. On further testing, the acidophilic material exhibited an orange colour on Congo Red staining, applegreen birefringence and light polarization (fig. 2). Staining was abolished by pre-treatment of the 

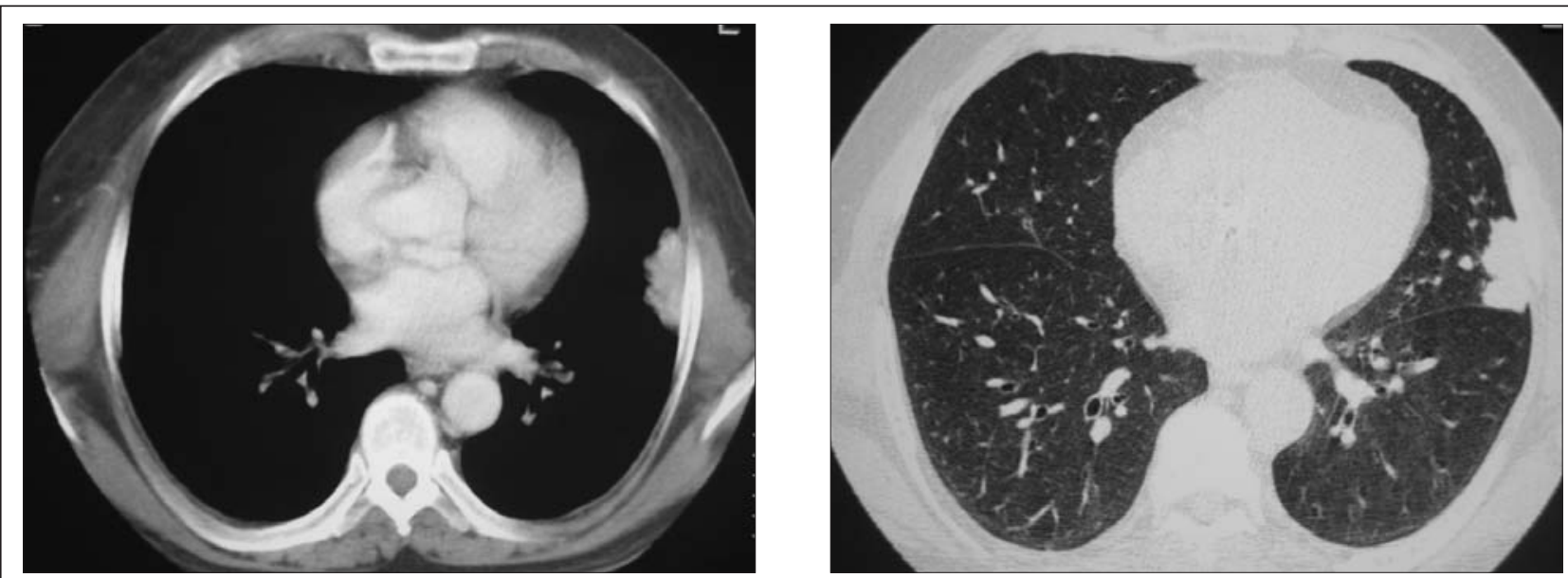

Fig. 1. - Pre-op. CT scan with iodized non ionic contrast medium showing a $3 \mathrm{~cm}$ in diameter nodular shadow in the upper segment of the lingula abutting the pleura, with irregular border and light hyperdensity.

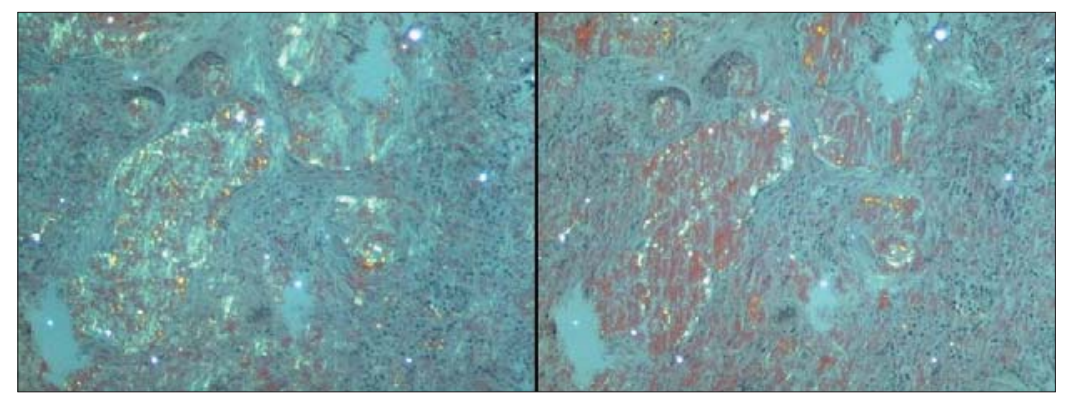

Fig. 2. - Nodular amyloid: a apple-green birefringence under polarizsed light, in Congo red stained section, it is shown (x 200).

section with potassium permanganate. Immunocytochemical staining using M 0759 - Monoclonal Mouse Anti-Human Amyloid A - was negative.

Due to the diagnosis of amyloidosis, a "work up" was then performed. Thyroid, heart and upper abdomen ultrasonographic examination was normal. Histological examination of gastric and bowel mucosa was negative for amyloid or active inflammatory disease.

After 8 months we operated him for an endoarteriectomy for a severe stenosis of the right internal carotid artery and at the 15 months follow up the patient was doing well, with no complaints and a negative chest-X-Ray.

\section{Discussion}

Amyloidosis is a group of diseases characterised by a deposition of insoluble protein fibrils, or proteins complex together with polysaccharide material in connective tissue, around parenchymal tissue cells and in the walls of blood vessels. The disease can be either localised (up to $20 \%$ of cases) or systemic (the remaining) [1-2-3].

The Systemic form is now classified into many different forms on the basis of the chemical nature of its amyloid precursor protein. Representative systemic amyloidosis consist of immunoglobulin light-chain (AL)-derived primary amyloidosis, reactive (secondary) AA amyloidosis, transthyretin (ATTR)-related hereditary amyloidosis and B2-microglobulin (Aß2M)-derived dialysis-related amyloidosis [1-2-3-10].
Pulmonary amyloid may be localised to the respiratory tract (with no systemic deposition of the amyloid) or may be part of a widespread process involving many organs.

There are three types of localized amyloidosis: tracheobronchial, diffuse interstitial and nodular parenchymal. This last one is an uncommon manifestation [1-2-3-10].

Amyloid nodules in the lung parenchyma are usually an incidental finding (on chest radiography or at autopsy) that needs to be distinguished (especially from neoplasia) [11-12-13].

They are usually peripheral and subpleural, occur more frequently in the lower lobes, may be bilateral, and range in diameter from $0.4 \mathrm{~cm}$ to 15 $\mathrm{cm}$. On the CT scan, they present almost all the characteristics of malignancy (irregular border, subpleurical extrication, hyperdensity after c.m. and so on), making a differential diagnosis difficult [12-13-14-15-16-17].

The lesions are difficult to diagnose (except by surgery or autopsy), as biopsy specimens are often insufficient for diagnosis in terms of both quantity and quality [12-18-19].

There are in fact two important factors to consider regarding biopsy method for suspected amyloid lesions. Firstly, amyloid is difficult to harvest because of its hard consistency. Secondly, although one malignant cell confirms malignancy, their absence cannot reliably exclude malignancy in most cases [12-18.19].

There are some "positive" reports about CTguided transbronchial biopsy, which appears to be more accurate than the CT-guided percutaneous needle biopsies. But this method is not always applicable, because of the nodular amyloidosis usually involved in the periphery of the lung and the subpleurical areas [20].

Open thoracotomy provides optimal access for all parenchymal lesions and permits simultaneous biopsy of other nodules. However, the course of a solitary parenchymal amyloid lesion is generally 
benign. Therefore it is rarely necessary to reset a nodular amyloid lesion unless it is causing respiratory symptoms. Nevertheless, histological diagnosis is necessary since, as already stated, a chest radiograph and a CT-scan cannot reliably differentiate an amyloid (benign) lesion from any other lesion (above all, malignant) [12-13-14-15-16].

The growing experience with video-assisted thoracoscopic procedures has led to combine diagnostic and therapeutic procedures at the same time. The minimally invasive surgery offers nearly $100 \%$ diagnostic accuracy, avoiding a delay in the diagnosis (especially with respect to potentially curable lung cancer), and offers an acceptable morbidity rate with virtually no associated mortality [4].

In the case we have presented, the definitive diagnosis was made possible only by a thoracoscopic approach and, in the meantime, it was therapeutic with no morbidity and/or mortality.

Somehow, the situation we present could be considered alongside other cases of coin lesions, suggesting a diagnosis of lung cancer where any attempt of diagnosis (through fiber optic bronchoscopy and/or fine needle aspiration) failed: the choice is almost always to undergo surgical diagnosis and treatment, unless the conditions of the patient are a contraindication of surgical intervention [4].

This report strongly highlights the importance of a video-assisted thoracoscopy (with all the familiar characteristics of the minimally invasive and "gentle" approach), as one of the main treatment options of indeterminate solitary pulmonary nodules in the outer third of the lung field.

Acknowledgment: The Authors want to thank Mrs Dynah Allardyce from Perth, West-Australia, for her linguistic review of the manuscript.

\section{References}

1. Gillmore JD, Hawkins PN. Amyloidosis and the respiratory tract. Thorax 1999; 54: 444-451.

2. Pepys MB. Amyloidosis. In: Weatherall DJ, Ledingham JGG, Warrell DA, eds. Oxford textbook of medicine, 3rd ed. Oxford: Oxford University Press, 1995; 1512-1524.

3. Utz JP, Swensen SJ, Gertz MA. Pulmonary amyloidosis: the Mayo Clinic experience from 1980-1993. Ann Intern Med 1996; 124: 407-413.
4. Liptay MJ. Solitary Pulmonary Nodule. Chest 1999; 116: 517S-518S.

5. Barcelo JR, Munoz A, Mane JM, Rubio I, Perez-Hoyos T, Viteri A, Lopez-Vivanco GM. Amyloidosis and lung cancer. Clin Lung Cancer 2003; 4: 249-51.

6. Dacic S, Colby TV, Yousem SA. Nodular amyloidoma and primary pulmonary lymphoma with amyloid production: a differential diagnostic problem. Mod Pathol 2000; 13: 934-40.

7. Slanetz PJ, Whitman GJ, Shepard JA, Chew FS. Nodular pulmonary amyloidosis. AJR 1994; 163: 296.

8. Kaw YT, Esparza AR. Solitary pleural amyloid nodules occurring as coin lesions diagnosed by fine-needle aspiration biopsy. Diagn Cytopathol 1991; 7: 304-307.

9. Vera-Alvarez J, Marigil-Gomez M, Abascal-Agorreta M, Pons-Bosque J. Localized pulmonary amyloidosis diagnosed by fine needle aspiration cytology. Acta Cytol 1993; 37: 846-8.

10. Shah PL, Gillmore JD, Copley SJ, et al. The importance of complete screening for amyloid fibril type and systemic disease in patients with amyloidosis in the respiratory tract. Sarcoidosis Vasc Diffuse Lung Dis 2002; 19: 134-42.

11. Howard ME, Ireton J, Daniels F, et al. Pulmonary presentations of amyloidosis. Respirology 2001; 6: 61-4.

12. Hui AN, Koss MN, Hochholzer L, et al. Amyloidosis presenting in the lower respiratory tract; clinicopathologic, radiologic,immunohistochemical, and histochemical studies on 48 cases. Arch Pathol Lab Med 1986; 110: 212-218.

13. Schulz C, Hauck RW, Nathrath WB, et al. Combined amyloidosis of the upper and lower respiratory tract. Respiration 1995; 62: 163-166.

14. Urban BA, Fishman EK, Goldman SM, et al. CT evaluation of amyloidosis: spectrum of disease. Radiographics 1993; 13: 1295-1308

15. Pickford HA, Swensen SJ, Utz JP. Thoracic cross-sectional imaging of amyloidosis. AJR 1997; 168: 351-5.

16. Gertz MA, Greipp PR. Clinical aspects of pulmonary amyloidosis. Chest 1986; 90: 790-1.

17. Kung J, Zhuang H, Yu JQ, Duarte PS, Alavi A. Intense fluorodeoxyglucose activity in pulmonary amyloid lesions on positron emission tomography. Clin $\mathrm{Nucl} \mathrm{Med}$ 2003; 28: 975-6.

18. Khoor A, Myers JL, Tazelaar HD, Kurtin PJ. Amyloidlike pulmonary nodules, including localized light-chain deposition: clinicopathologic analysis of three cases. Am J Clin Pathol 2004; 121: 200-4.

19. Mollers MJ, van Schaik JP, van der Putte SC. Pulmonary amyloidoma. Histologic proof yielded by transthoracic coaxial fine needle biopsy. Chest 1992; 102: $1597-8$.

20. Kitamura H, Kobayashi T, Kaneko $\mathrm{M}$ at al. Pulmonary Amyloidosis diagnosed by CT-guided transbronchial biopsy: a case report. Jap J Clin Oncol 2001; 31: 209211. 